SLAC-WP-050

May 2005

\title{
Quantum Coherence Arguments for Cosmological Scale
}

\author{
James Lindesay
}


4 May 2005

\title{
Quantum Coherence Arguments for Cosmological Scale
}

\author{
James Lindesay \\ Stanford Linear Accelerator Center \\ Howard University
}

Work supported by Department of Energy contract DE--AC02--76SF00515 


\section{Abstract}

Homogeneity and correlations in the observed CMB are indicative of some form of cosmological coherence in early times. Quantum coherence in the early universe would be expected to give space-like phase coherence to any effects sourced to those times. If dark energy de-coherence is assumed to occur when the rate of expansion of the relevant cosmological scale parameter in the Friedmann-Lemaitre equations is no longer supra-luminal, a critical energy density is immediately defined. It is shown that the general class of dynamical models so defined necessarily requires a spatially flat cosmology in order to be consistent with observed structure formation. The basic assumption is that the dark energy density which is fixed during de-coherence is to be identified with the cosmological constant. It is shown for the entire class of models that the expected amplitude of fluctuations driven by the dark energy de-coherence process is of the order needed to evolve into the fluctuations observed in cosmic microwave background radiation and galactic clustering. The densities involved during de-coherence which correspond to the measured dark energy density turn out to be of the electroweak symmetry restoration scale. In an inflationary cosmology, this choice of the scale parameter in the FL equations directly relates the scale of dark energy decoherence to the De Sitter scales (associated with the positive cosmological constants) at both early and late times. 


\section{Outline of Presentation}

I. Cosmic Microwave Background Radiation

II. Macroscopic Quantum Systems

III. Friedmann-LeMaitre Dynamics

IV. Zero-Point Motions

V. Dark Energy Partitions and Fluctuations

VI. Thermalization Scenarios

VII. Discussion and Conclusions 


\section{COBE CMB Probe}

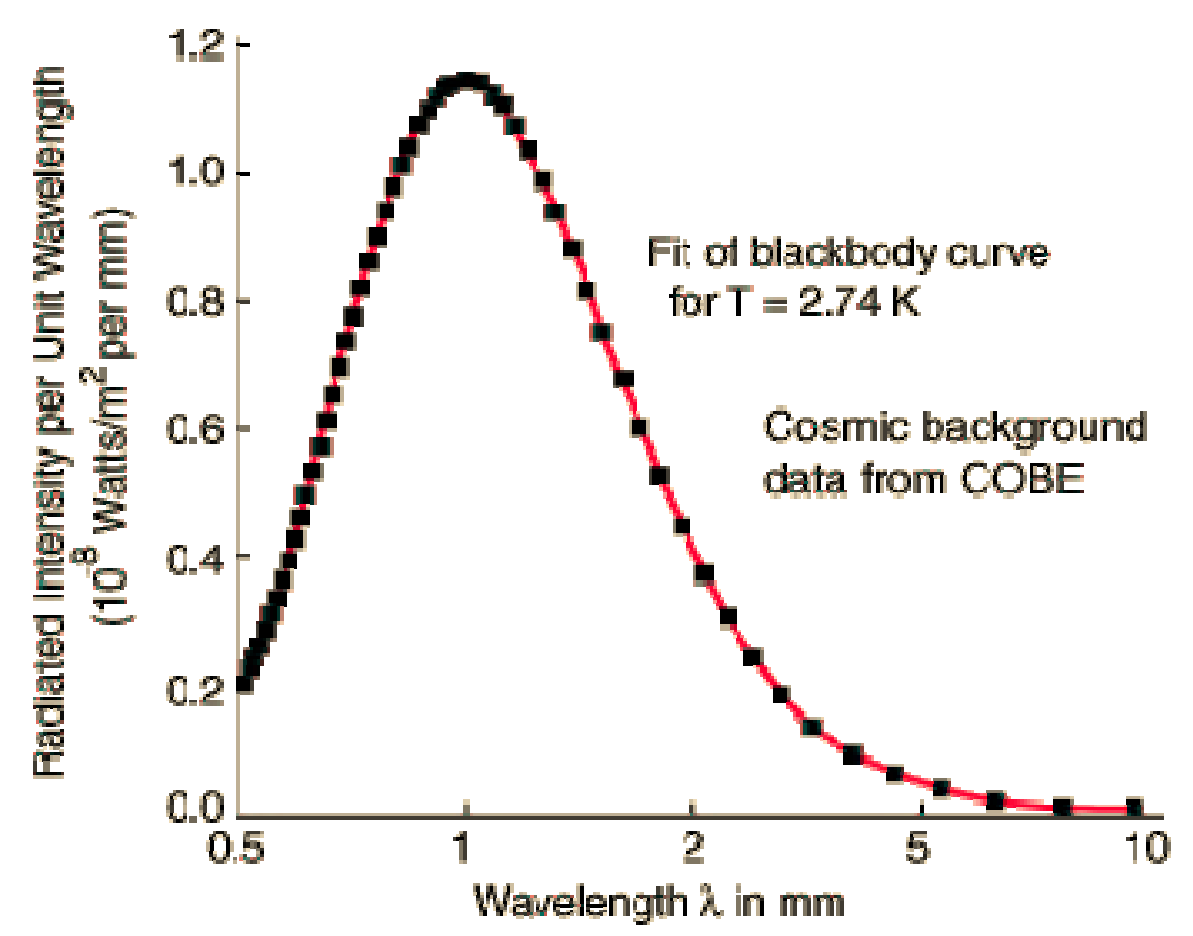




\subsection{K Cosmic Microwave Background}

$4 \pi$ true view of sky without enhanced contrast 


\section{$369 \mathrm{~km} / \mathrm{sec}$ Dipole pattern}

(Contrast times 400)

Temperature variation of plus/minus $0.00335 \mathrm{~K}$ 


\title{
CMB Contrast times 2000
}

\author{
Variation due to Milky Way Galaxy
}




\section{Remnant of the BIG BANG}

Contrast $=30,000$ times 


\section{Fluctuations}

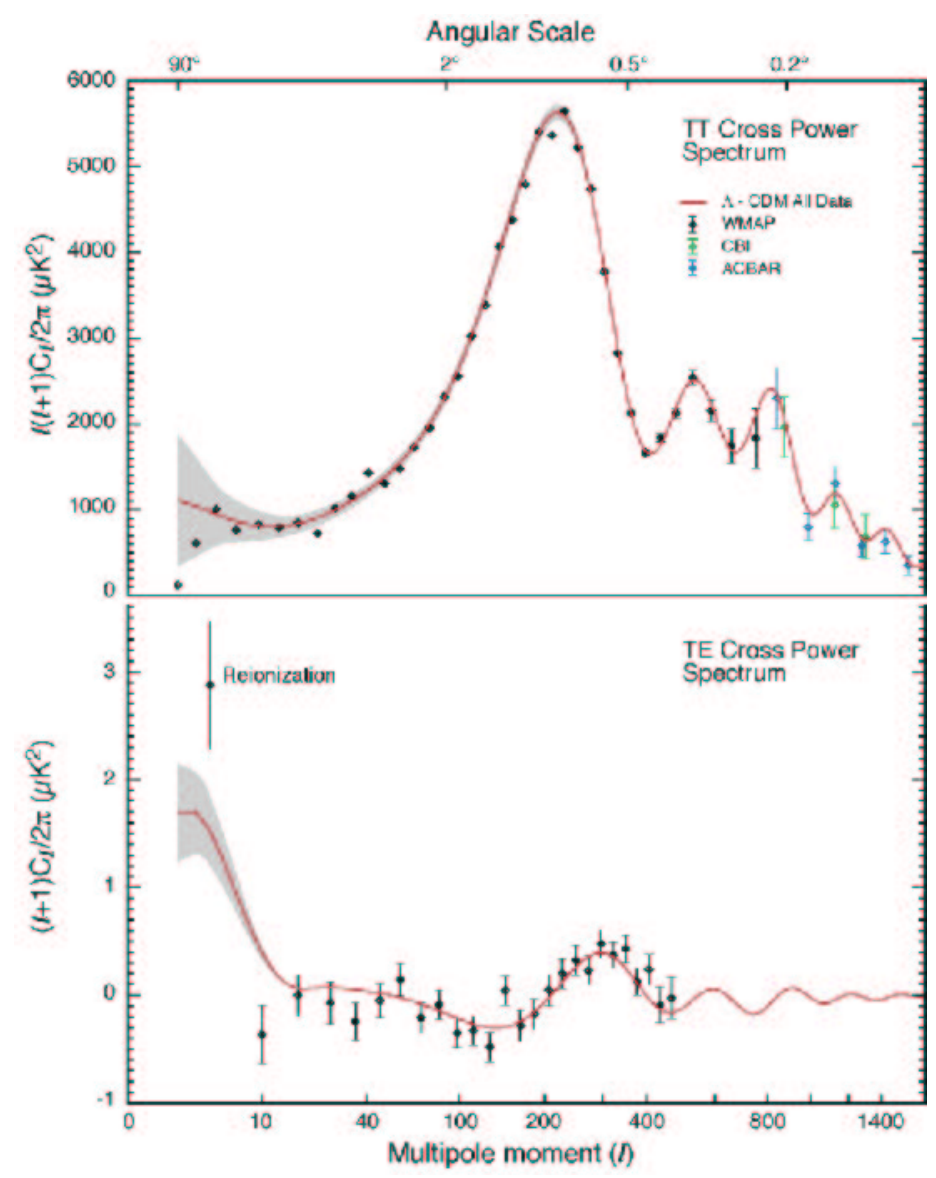

Observed Power Spectrum 


\section{The Horizon Problem}

A. Why is the Cosmic Microwave Background so uniform?

B. How can there be space-like correlations in the fluctuations and phase?

OUR APPROACH: At sometime in the past, the whole cosmology had global quantum coherence.

We will extrapolate our present understanding of physics backwards until we have trouble with basic concepts. 


\section{Quantum Basics}

Space-Like Correlations: Vacuum expectation values of the symmetric sum of a field operator at different points is non-vanishing for space-like related events

$$
\begin{gathered}
<v a c|\phi(x) \phi(y)+\phi(y) \phi(x)| v a c>=\frac{1}{4 \pi^{2} s^{2}} \\
s^{2}=|\vec{x}-\vec{y}|^{2}-\left(x^{0}-y^{0}\right)^{2}
\end{gathered}
$$

Since the vacuum expectation value of the field is expected to vanish, this requires spacelike correlations

$$
\begin{aligned}
& <v a c|\phi(x) \phi(y)+\phi(y) \phi(x)| v a c>\neq \\
& 2<v a c|\phi(x)| v a c><v a c|\phi(y)| v a c>
\end{aligned}
$$

However, the commutator of the field does vanish for spacelike separations, preventing measurement at $y$ from changing the probability distribution at $\mathrm{x}$. 


\section{Quantum Measurement}

The Triangle Inequality: A fundamental limit is placed on quantum measurements due to non-commutivity of operations

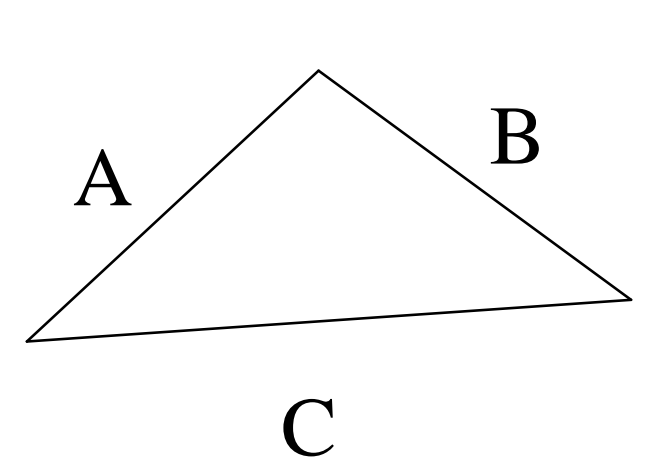

$$
\begin{aligned}
& \mathrm{C}=\mathrm{A}+\mathrm{B}, \quad|\mathrm{C}| \leq|\mathrm{A}|+|\mathrm{B}| \\
& |\mathrm{A}||\mathrm{B}| \geq|[\mathrm{A}, \mathrm{B}]| / 2 \\
& \text { Let } \mathrm{A}=\mathrm{X}-\langle\mathrm{X}\rangle, \quad \mathrm{B}=\mathrm{P}-\langle\mathrm{P}\rangle \\
& \Rightarrow \Delta X \Delta P \geq \frac{\hbar}{2}
\end{aligned}
$$




\section{About Zero-Point Motions}

- Zero-point motion of sources correspond to vacuum energy of the associated quanta.

- Physical effects associated with the zero point motions do not depend on the coupling constant of the involved interactions, but do depend on the nature of the interaction.

- In physical systems, zero-point motions correspond to saturation of the triangle inequality. 


\section{Coherence Length Scales}

Zero-point motions

$$
\Delta P \Delta X=\frac{\hbar}{2}
$$

Macroscopic
quantum $k_{B} T_{\text {crit }} \approx$ thermal energies $\approx \sqrt{(\Delta P)^{2}+m^{2}}-m \approx \frac{\hbar c}{\xi_{\text {thermal }}}$ systems

$$
\Rightarrow \xi_{\text {thermal }} \lambda_{m} \approx 8(\Delta X)^{2}
$$

General zero-point motions

$$
\frac{(\Delta P)^{2}}{2 m}=\varepsilon \equiv \frac{\hbar c}{R_{\varepsilon}} \Rightarrow R_{\varepsilon} \lambda_{m}=8(\Delta X)^{2}
$$

Degenerate Fermi gas

$$
n=\frac{g_{m}}{6 \pi^{2}}\left(2 m \varepsilon_{\text {thermal }}\right)^{3 / 2} \Rightarrow R_{\varepsilon} \lambda_{m}=\frac{1}{2}\left(\frac{6 \pi^{2}}{g_{m}}\right)^{2 / 3}(\Delta X)^{2}
$$




\section{Macroscopic Quantum Systems}

- For ${ }^{4} \mathrm{He}$, atomic spacing, and low particle mass give comparable zero-point energies to the weak inter-atomic forces

- The Casimir effect gives measured effects due to vacuum energy and geometry

- The Casimir effect is equivalent to energies produced by zeropoint motions of the sources at absolute zero

- For gravitating masses within their Compton wavelength, we expect significant zero-point motions

$$
\begin{gathered}
\Delta p \Delta R>\hbar \rightarrow K E \sim \frac{(\Delta p)^{2}}{2 m} \sim \frac{\hbar^{2}}{2 m \bar{V}^{2 / 3}} \\
E_{\text {vacuum }}=\sum_{\text {modes }} \frac{1}{2} \hbar \omega_{k} \\
\frac{\Delta E_{\text {vacuum }}^{\text {plates }}}{\text { Area }}=-\frac{\pi^{2}}{720} \frac{\hbar c}{a^{3}} \\
\Delta E_{\text {vacuum }}^{\text {sphere }}=+0.92353 \frac{\hbar c}{a} \\
E_{o} \sim \sqrt{\left(\frac{\hbar c}{\lambda_{m}}\right)^{2}+\left(m c^{2}\right)^{2}} \sim \sqrt{2} m c^{2} \\
V \sim-\frac{G_{N} m^{2}}{\lambda_{m}} \sim \frac{m^{2}}{M_{P}^{2}} m c^{2}<<E_{o}
\end{gathered}
$$




\section{An Example: Cold Dark Bosonic Matter}

For non-relativistic particulate bosonic dark matter, the relationship between number density and critical density for a free bose gas is given by

$$
\begin{gathered}
\frac{N_{D M}}{V}=\frac{\zeta(3 / 2) \Gamma(3 / 2)}{(2 \pi)^{2} \hbar^{3}}\left(2 m_{D M} k_{B} T_{c r i t}\right)^{3 / 2} \quad, \quad \rho_{D M} \cong \frac{N_{D M}}{V} m_{D M} c^{2} \\
k_{B} T_{c r i t}=\frac{1}{2} \frac{\rho_{D M}^{2 / 3}}{\left(m_{D M} c^{2}\right)^{5 / 3}} \frac{(2 \pi)^{2}(\hbar c)^{3}}{\zeta(3 / 2) \Gamma(3 / 2)} \cong 1.69 \times 10^{-31} \mathrm{GeV} \frac{z^{2}}{g_{m_{D M}}^{2 / 3}}\left(\frac{G e V}{m_{D M} c^{2}}\right)^{5 / 3}
\end{gathered}
$$

The temperature of the photon gas is expected to scale with the redshift when appropriate pair creation threshold effects are properly incorporated. Setting critical temperature the same as the photon temperature

$$
\begin{aligned}
z_{c r i t} & =\left(\frac{g\left(T_{\gamma o}\right)}{g\left(T_{c r i t}\right)}\right)^{1 / 3} \frac{2 g_{m_{D M}}}{(\hbar c)^{2}}\left(\frac{\left(k_{B} T_{\gamma_{o}}\right)^{3 / 2}}{\rho_{D M}} \frac{\zeta(3 / 2) \Gamma(3 / 2)}{(2 \pi)^{2}}\right)^{2 / 3}\left(m_{D M} c^{2}\right)^{5 / 3} \\
& \cong 1.38 \times 10^{18}\left(\frac{g\left(T_{\gamma o}\right)}{g\left(T_{c r i t}\right)}\right)^{1 / 3} g_{m_{D M}}^{2 / 3}\left(\frac{m_{D M} c^{2}}{G e V}\right)^{5 / 3}
\end{aligned}
$$

where $g(z)$ counts the number of low mass degrees of freedom available at redshift $z$. Transition is non-relativistic $\Rightarrow m_{D M}>\frac{15 e V}{g_{m_{D M}}}$
if $T_{\text {crit }}<m_{D M}$ 


\section{Particulate Dark Matter/Photon Ratio}

Adiabatic expansion is expected to preserve the ratio of particulate dark matter number to photon number.

Relating photon energy density to number density gives

$$
\begin{aligned}
\frac{N_{D M}}{N_{\gamma}} & =\frac{\zeta(4) \Gamma(4)}{\zeta(3) \Gamma(3)}\left(\frac{\Omega_{D M o}}{\Omega_{\gamma o}}\right) \frac{k_{B} T_{\gamma o}}{m_{D M} c^{2}} \\
& \cong 3.70 \times 10^{-9}\left(\frac{G e V}{m_{D M} c^{2}}\right)
\end{aligned}
$$

This should give a phenomenological handle on the thermalization process for the dark matter. 


\section{De-Coherence Due to Microscopic Scale}

- One expects a quantum phase change occurs when the energy density of the expanding cosmology reaches a critical density within a region of coherence $\lambda_{\mathrm{m}}$.
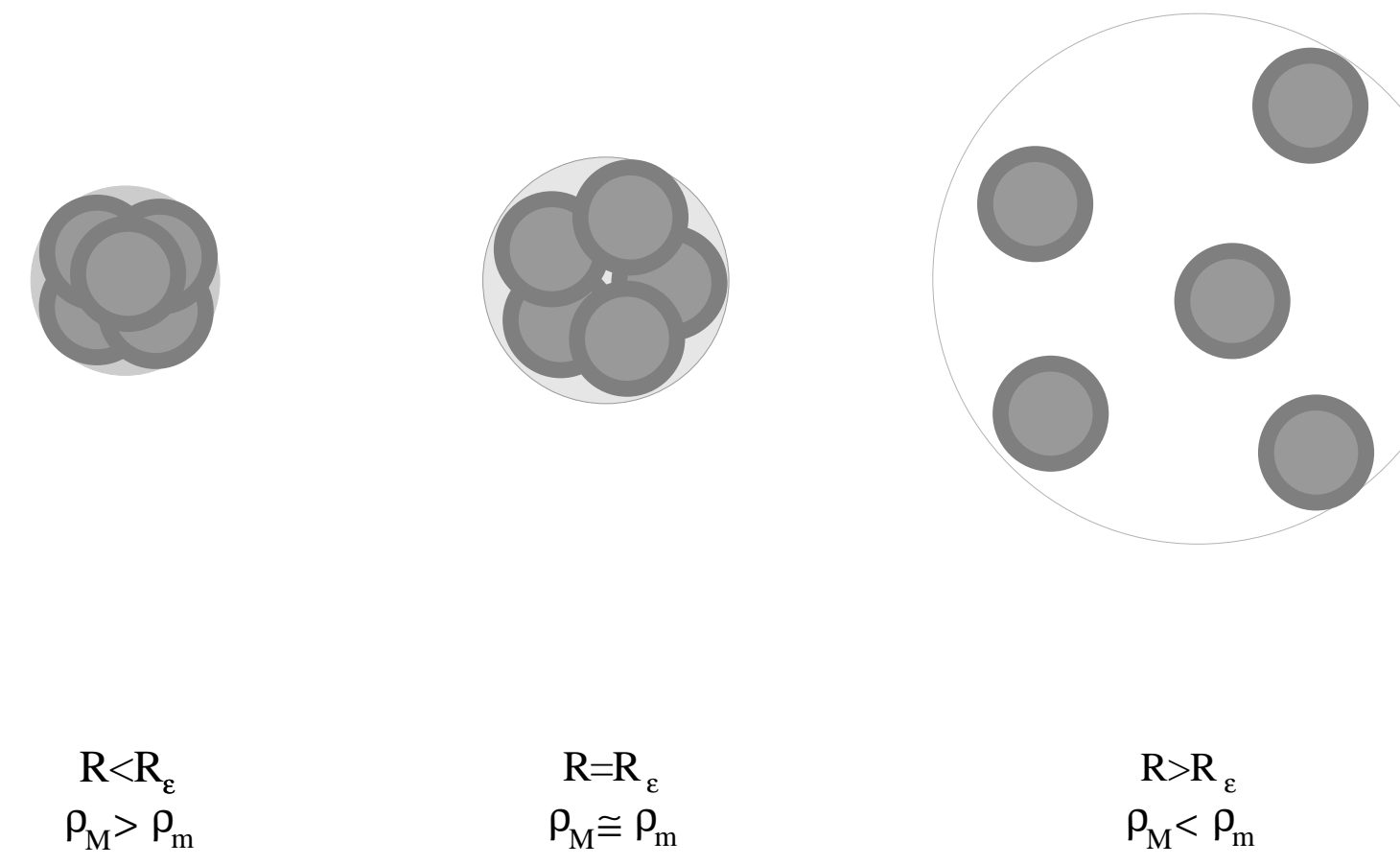

$$
\begin{gathered}
\mathrm{R}=\mathrm{R}_{\varepsilon} \\
\rho_{\mathrm{M}} \cong \rho_{\mathrm{m}}
\end{gathered}
$$$$
\begin{gathered}
\mathrm{R}>\mathrm{R}_{\varepsilon} \\
\rho_{\mathrm{M}}<\rho_{\mathrm{m}}
\end{gathered}
$$ 


\section{Einstein's Equation}

- Experiments by Overhauser, et.al. have demonstrated gravitation of coherent systems

- We will assume that Einstein's equation describes the evolution of the cosmology during the epoch of interest (post-decoherence)

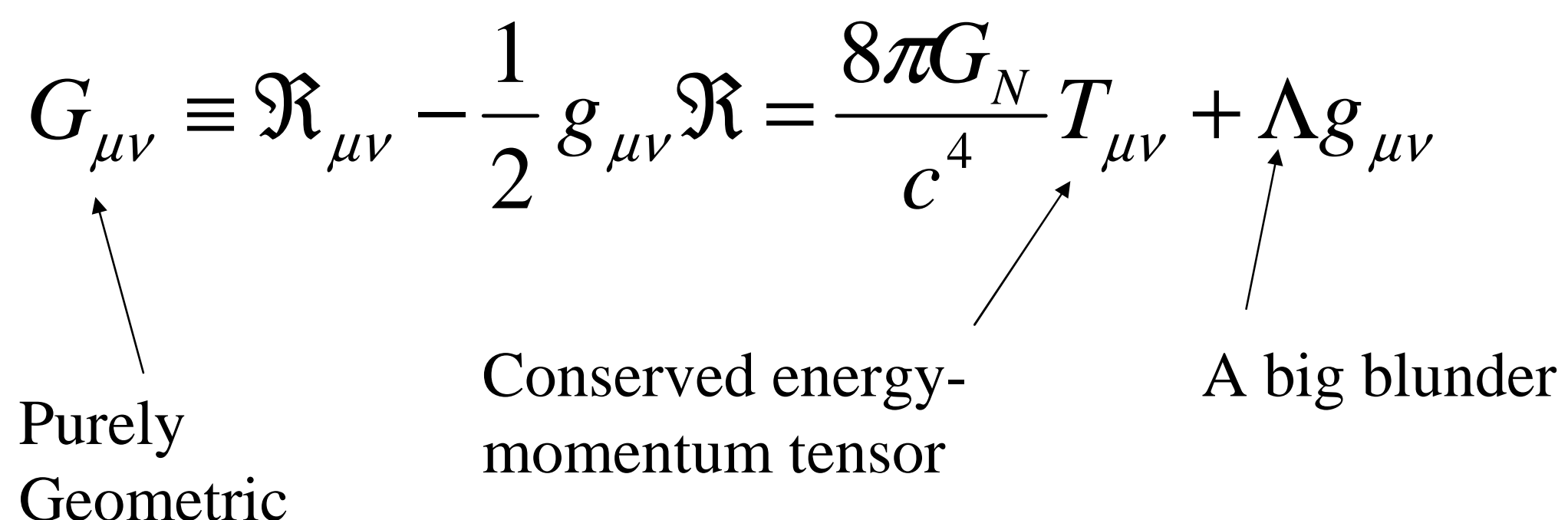

Geometric 


\section{Cosmological Constant}

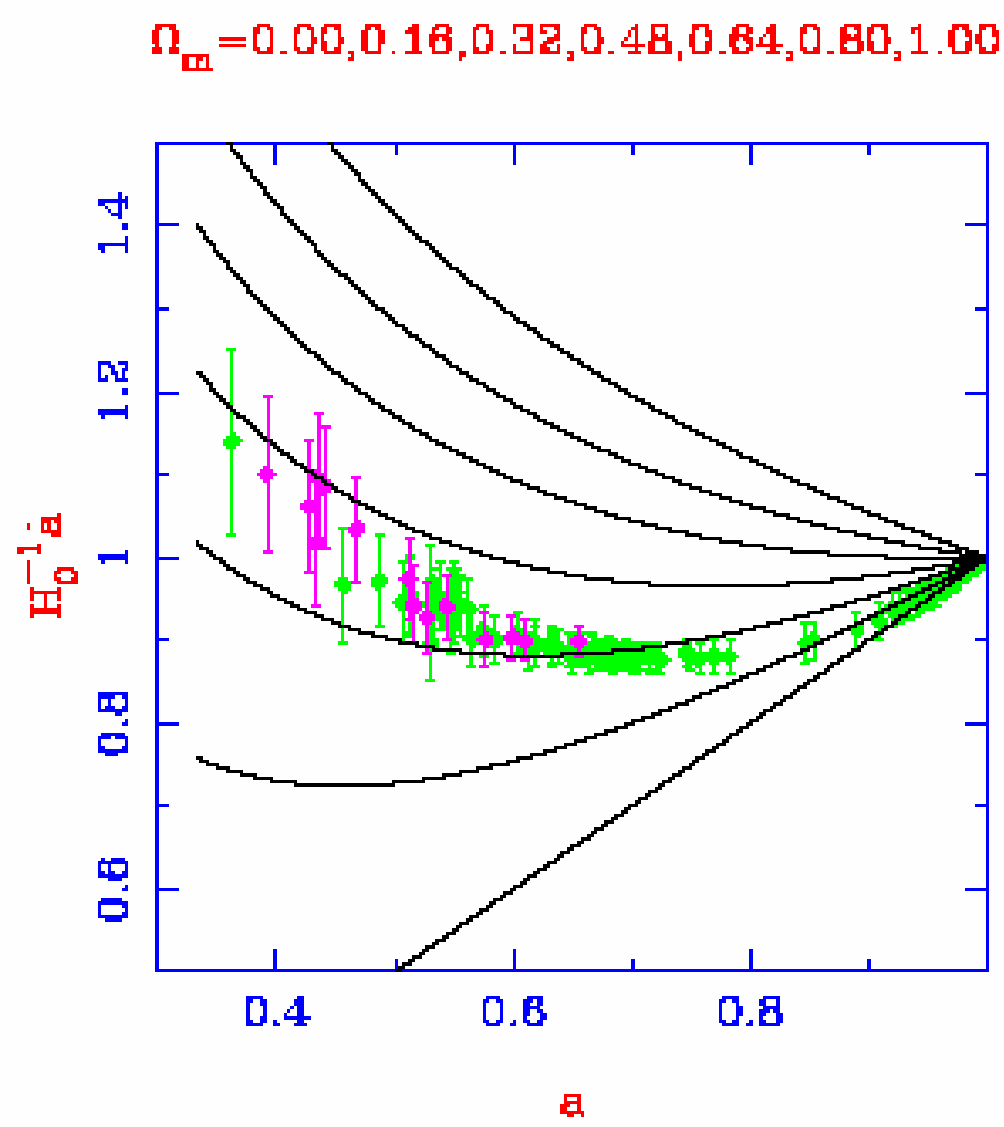

Observed deceleration followed by acceleration in late times is best fit by a cosmological constant. 


\section{Friedmann-Robertson-Walker-Lemaitre Cosmology}

- Assume a spatially homogeneous and isotropic cosmology (The cosmological principle) $\rightarrow$ FRW metric

$$
d s^{2}=-c^{2} d t^{2}+R^{2}(t)\left(\frac{d r^{2}}{1-k r^{2}}+r^{2} d \vartheta^{2}+r^{2} \sin ^{2} \vartheta d \varphi^{2}\right)
$$

- Assume an ideal fluid as the form for the energymomentum tensor in Einstein's equation $\rightarrow$ FL equations

$$
\begin{aligned}
& \left(\frac{\dot{R}}{R}\right)^{2}=\frac{8 \pi G_{N}}{3}\left(\rho+\rho_{\Lambda}\right)-\frac{k}{R^{2}} \\
& \frac{\ddot{R}}{R}=-\frac{4 \pi G_{N}}{3}\left(\rho+3 P-2 \rho_{\Lambda}\right)
\end{aligned}
$$




\section{Zero-Point Motions}

Uncertainty for quantum energy scale $\quad \Delta E \Delta t \geq \frac{\hbar}{2} \quad, \quad \Delta E \equiv \varepsilon \equiv \frac{\hbar c}{R_{\varepsilon}}$

Radiation dominated cosmology

$$
\frac{R_{\varepsilon}}{R_{D C}}=\sqrt{\frac{t_{\varepsilon}}{t_{D C}}} \quad, \quad \dot{R}_{D C} \equiv c \quad, \quad t_{D C} \equiv \frac{R_{D C}}{2 c}
$$

F-L Equation

$$
\left(\frac{\dot{R}}{R}\right)^{2} \cong \frac{8 \pi G_{N}}{3 c^{4}} \rho_{D C} \frac{R_{D C}^{4}}{R^{4}}=\frac{c^{2} R_{D C}^{2}}{R^{4}} \Rightarrow \frac{\dot{R}}{c}=\frac{R_{D C}}{R}
$$

$$
\Delta t_{\varepsilon} \cong t_{D C}\left(\frac{R_{\mathcal{\varepsilon}}}{R_{D C}}\right)^{2} \quad, \quad \Delta E \Delta t_{\varepsilon} \cong \frac{\hbar}{2} \frac{c}{\dot{R}_{\varepsilon}} \Rightarrow \dot{R}_{\varepsilon} \leq c
$$

De-coherence

Null geodesic $\quad c d t=R d \chi \quad, \quad \Delta \chi_{D C}=\int_{0}^{t_{D C}} \frac{c d t^{\prime}}{R\left(t^{\prime}\right)}=1$ Criterion

(Radiation dominated only)

$$
D_{\text {Horizon }}=R_{D C} \Delta \chi_{D C}=R_{D C}
$$




$$
\begin{aligned}
& \text { Spatial Flatness } \\
& \left(\frac{\dot{R}}{R}\right)^{2}=\frac{8 \pi G_{N}}{3 c^{2}}\left(\rho+\rho_{\Lambda}\right)-\frac{k c^{2}}{R^{2}}
\end{aligned}
$$

An open universe with $\mathrm{k}=-1$ is excluded from undergoing the de-coherence transition due to the dark energy term. A closed universe with $\mathrm{k}=+1$ never expands enough for structure to form.

$$
0=\frac{8 \pi G_{N}}{3}\left(\rho\left(R_{\max }\right)+\rho_{\Lambda}\right)-\left(\frac{c}{R_{\max }}\right)^{2} \Rightarrow R_{\max }^{2} \cong 2 R_{D C}^{2}
$$

For a flat cosmology, the density at de-coherence is given by

$$
\rho_{D C} \equiv \frac{3 c^{2}}{8 \pi G_{N}}\left(\frac{c}{R_{D C}}\right)^{2}-\rho_{\Lambda}
$$




\section{De-Coherence Extrapolation}

If the cosmology remains radiation dominated in the standard way down to $t=0$, then the scale parameter satisfies

$$
R(t)=\left(\frac{t}{t_{D C}}\right)^{1 / 2} \Rightarrow t_{D C}=\frac{R_{D C}}{2 c}
$$

Using the FL densities at radiation-matter (dust) equality $\rho_{M}\left(z_{e q}\right)=\rho_{\text {rad }}\left(z_{e q}\right)$ one can extrapolate back to the de-coherence period. Ignoring threshold effects (which give small corrections near particle thresholds while they are nonrelativistic), this gives

$$
1+z_{D C}=\left(\frac{\rho_{D C}}{\rho_{M o}}\left(1+z_{e q}\right)\right)^{1 / 4} \cong\left(\frac{c}{H_{o} R_{D C}}\right)^{1 / 2}\left(\frac{1+z_{e q}}{\Omega_{M o}}\right)^{1 / 4}
$$

Here, $\Omega_{\mathrm{Mo}}$ is the present normalized non-relativistic mass density. The scale parameter at the present time is then expressed in terms of this redshift using the usual definition

$$
R_{o}=\left(1+z_{D C}\right) R_{D C}
$$




\section{Scale Evolution}

The evolution of the cosmology during the period for which the dark energy density is de-coupled from the FL energy density is modeled using the FL equations. There is a period of deceleration, followed by acceleration towards a De Sitter expansion. The rate of scale parameter expansion is sub-luminal during a finite period of this evolution, as shown in the Figures.
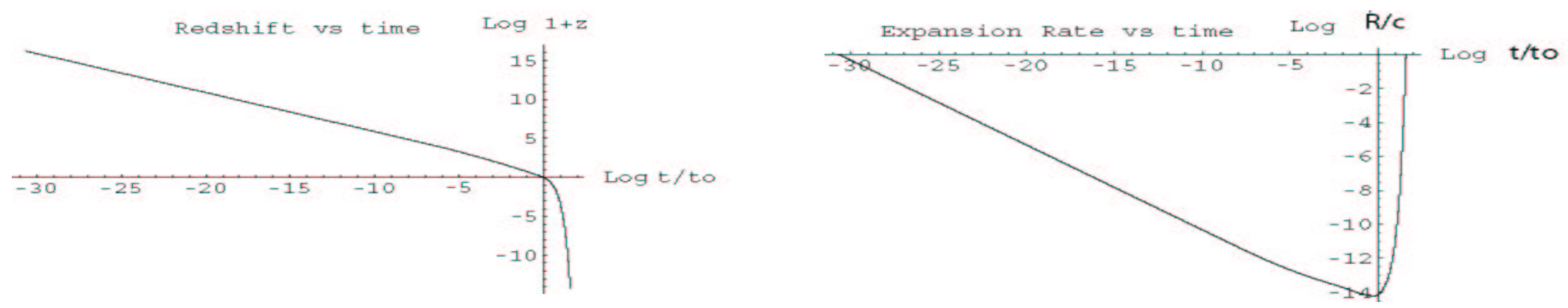

The particular value for the scale at de-coherence is determined by the microscopic makeup of the dark energy. Present time corresponds to the origin on both graphs. The value of the expansion rate is $\mathrm{c}$ for $R=R_{D C}$, as well as when the expansion scale reaches the De Sitter radius

$$
R_{\Lambda} \equiv \sqrt{\frac{3}{\Lambda}} \approx 10^{28} \mathrm{~cm} \approx 1.6 \times 10^{10} \mathrm{ly}
$$




\section{More on Zero-Point Motions}

- We will assume that the cosmological vacuum energy is due to the zero point motions just at the availability of luminal equilibrations

$$
\rho_{\Lambda}=\frac{\varepsilon}{\left(R_{\varepsilon}\right)^{3}}=\frac{\varepsilon^{4}}{(\hbar c)^{3}} \quad \varepsilon \cong 2.37 \times 10^{-12} \mathrm{GeV}
$$

This means that the cosmological constant is fixed by the physical condition of luminal expansion rate (and the associated de-coherence) being met. At this stage no further assumption of microscopic scale will be made. We will construct the mass units responsible. 


\section{Cosmological Energy Density}

Energy per particle $\quad$ mass $=M \quad, \quad \widetilde{\varepsilon} \equiv \frac{\varepsilon}{N} \cong \frac{(\Delta P)^{2}}{2 M}=\frac{\hbar^{2}}{8 M(\Delta X)^{2}}$

Require that momentum coherence scale is same as energy coherence scale

Masses undergoing zero-point motions

Expected energy density

$$
\widetilde{\varepsilon}=\frac{\varepsilon}{N} \cong \frac{\varepsilon^{2}}{8 M c^{2}} \Rightarrow M c^{2} \cong \frac{1}{8} N \varepsilon
$$

$$
\begin{aligned}
& \text { If } \rho=\rho_{D C}=\frac{3}{8 \pi} \frac{\left(M_{P} c^{2} \varepsilon\right)^{2}}{(\hbar c)^{3}}\left(\cong 9.97 \times 10^{13} G e V^{4}\right) \Rightarrow N \cong \sqrt{\frac{3}{\pi}} \frac{M_{P} c^{2}}{\varepsilon} \\
& M \cong \frac{1}{8} \sqrt{\frac{3}{\pi}} M_{P} \quad \begin{array}{l}
\text { Planck Mass scale units are undergoing the } \\
\text { zero-point motions. } \varepsilon \text { is still arbitrary! }
\end{array}
\end{aligned}
$$

If space-time were Schwarzschild, the density

is about that associated with a black hole

$$
\frac{R_{S}}{R_{D C}} \sim 1
$$




\section{Dark and Cosmological Energy}

Friedmann-Lemaitre Equation $\quad\left(\frac{\dot{R}}{R}\right)^{2}=\frac{8 \pi G_{N}}{3}\left(\rho+\rho_{\Lambda}\right)$

Dark energy de-coherence

$$
\begin{aligned}
& \left(\frac{c}{R_{\varepsilon}}\right)^{2}=\frac{8 \pi G_{N}}{3}\left(\rho_{\varepsilon}+\rho_{\Lambda}\right) \\
& =\frac{8 \pi G_{N}}{3}\left(N_{\varepsilon}+\frac{1}{2}\right) \underbrace{\frac{\hbar \omega_{\varepsilon}}{R_{\varepsilon}^{3}} \equiv \frac{8 \pi G_{N}}{3}\left(2 N_{\varepsilon}+1\right) \varepsilon^{4}}_{\text {collective mode quanta }}
\end{aligned}
$$$$
\text { condition }
$$

(gravons) in the de-coherent mode

De-coherent energy density for quantized energy mode $\varepsilon$

$$
\rho_{\varepsilon} \equiv \frac{3}{8 \pi}\left(M_{P} c^{2} \varepsilon\right)^{2}=2 N_{\varepsilon} \varepsilon^{4} \cong \frac{1}{8} N^{2} \varepsilon^{4}
$$

$\mathrm{N}_{\varepsilon}$ is the number of "gravons", $\mathrm{N}$ is the number of coherent Planck mass units. 


\section{Why Does Dark Energy Freeze Out?}

-The coherent collective modes of Planck mass units have been referred to as gravons.

-The zero point motions of these coherent Planck mass sized units have been shown to correspond to the vacuum energy of the gravons.

-After de-coherence, the coherence scale changes from $\mathrm{R}_{\varepsilon}$ to microscopic scale $\lambda_{\mathrm{m}}$ or $\Delta \mathrm{x} \ll \mathrm{R}_{\varepsilon}$ (ie a phase transition occurs).

$$
\begin{aligned}
& 2 N_{\varepsilon} \varepsilon^{4} \Rightarrow \rho_{D C} \equiv \rho_{\varepsilon} \longleftarrow \text { Friedmann-LeMaitre energy density } \\
& \varepsilon^{4} \Rightarrow \rho_{\Lambda} \quad \longleftarrow \text { Dark energy density }
\end{aligned}
$$

The vacuum energy of the gravons (or zero point motions of the coherent Planck scale mass units) de-cohere from the FL energy density, microscopically freezing this out as dark energy. 


\section{Statistical Dark Energy Partitions}

Vacuum energy density physically manifests in the Casimir effect. Lifshitz and his collaborators demonstrated that the Casimir force can be thought of as the superposition of the van der Waals attractions between individual molecules that make up the attracting media resulting from the zero-point motions of the sources. Since these motions are inherently a quantum effect for systems which manifest vacuum energy, one expects space-like correlations consistent with a quantum phenomenon.

A weakly interacting sea of the quantum fluctuations due to zero point motions should establish statistical variations in this "dark energy" density during de-coherence. One should be able to use simple counting arguments to quantify these variations. If the zero-point motions of the sources have a statistical weight $\Omega\left(E_{B}\right)$ associated with a partition $B$ having energy $E_{B}$ while holding total energy fixed, then the probability of such a partitioning is given by

$$
P\left(E_{B}\right)=\frac{\Omega\left(E_{B}\right)}{\Omega_{t o t}}=\frac{\Omega_{B}\left(E_{B}\right) \Omega_{\bar{B}}\left(E_{t o t}-E_{B}\right)}{\Omega_{t o t}}
$$

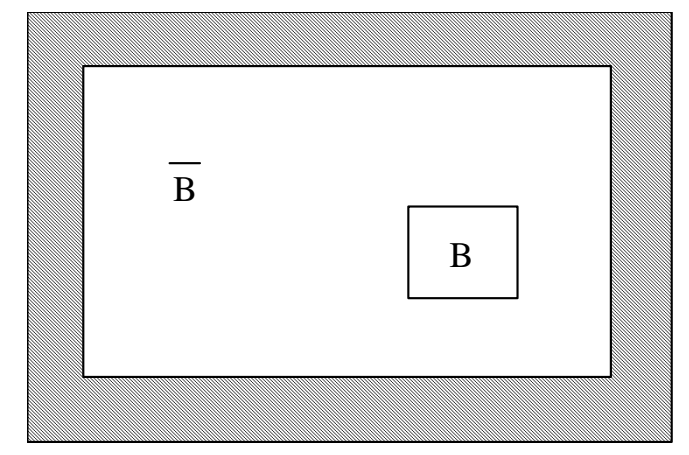

where $\bar{B}$ represents all external to the $B$ partition. 


\section{Statistics of Fluctuations}

Requiring that the most likely configuration of energy partitions results when (the log of) this probability is maximized, this distribution gives a uniform dark energy distribution if

$$
\frac{1}{E_{\Lambda}} \equiv \frac{d}{d E_{B}} \log \Omega\left(E_{B}\right)
$$

(zeroth law of thermodynamics) Here $E_{A}$ is an intensive energy associated with the statistical bath and boundary conditions. If one next examines a "small" partition $B$ for which the reservoir has energy $E_{\text {tot }}-E_{B}$, one can examine the (log of the) lowest order fluctuations from the reservoir to show $\Omega_{\bar{B}}\left(E_{t o t}-E_{B}\right) \cong \Omega_{\bar{B}}\left(E_{t o t}\right) e^{-E / E_{\Lambda}}$ thus defining a probability distribution

$$
P(E)=\frac{e^{-E^{\prime} / E_{\curlywedge}}}{\sum_{E} e^{-E^{\prime} / E_{\Lambda}}}
$$

For such an ensemble, the fluctuations satisfy the usual relation

$$
\left\langle(\delta E)^{2}\right\rangle=E_{\Lambda}^{2} \frac{d}{d E_{\Lambda}}\langle E\rangle
$$




\section{Equation of State}

A typical equation of state will connect the extensive variable $<E>$ to a dimensionless extensive variable that counts the available degrees of freedom $N_{D o F}$. A general equation of state satisfies

$$
\Rightarrow \frac{\left\langle(\delta E)^{2}\right\rangle}{\langle E\rangle^{2}}=a \frac{E_{\Lambda}}{E} \sim \frac{1}{N_{D o F}} \quad \begin{aligned}
& \text { e is an internal } \\
& \text { energy scale }
\end{aligned}
$$

In terms of the densities, one can directly write

$$
\frac{\left\langle(\delta E)^{2}\right\rangle}{\langle E\rangle^{2}}=\frac{\left\langle(\delta \rho)^{2}\right\rangle}{\langle\rho\rangle^{2}} \sim \frac{\rho_{\Lambda}}{\rho}
$$

This means that the amplitude of relative fluctuations $\delta \rho / \rho$ is expected to be of the order

$$
\Delta \equiv\left(\frac{\rho_{\Lambda}}{\rho}\right)^{1 / 2}
$$




\section{Pre-coherence and De-coherence}

- We define dark energy de-coherence to occur when the relevant FRW expansion rate is c. We have found this to be most consistent with a flat space.

$$
\left(\frac{c}{R_{\varepsilon}}\right)^{2}=\frac{8 \pi G_{N}}{3}\left(\rho_{D C}+\rho_{\Lambda}\right) \cong \frac{8 \pi G_{N}}{3} \rho_{D C}
$$

- This allows us to calculate the temperature of a thermal system at de-coherence as (or into which) the FL energy density thermalizes.

$$
\rho_{D C}=g\left(T_{\varepsilon}\right) \frac{\pi^{2}}{30} \frac{\left(k_{B} T_{\varepsilon}\right)^{4}}{(\hbar c)^{3}}
$$




\section{Fluctuations}

- The quantum fluctuations which become manifest during de-coherence are driven by the vacuum energy. This gives the scale of the amplitude of density fluctuations as

$$
\left.\frac{\delta \rho}{\rho}\right|_{\text {de-coherence }} \sim\left(\frac{\rho_{\Lambda}}{\rho_{D C}}\right)^{1 / 2} \sim\left(\frac{1}{N_{D o F}}\right)^{1 / 2}
$$

- Recall that the red shift at de-coherence is

$$
1+z_{D C}=\left(\frac{\rho_{D C}}{\rho_{M o}}\left(1+z_{e q}\right)\right)^{1 / 4} \cong\left(\frac{c}{H_{o} R_{D C}}\right)^{1 / 2}\left(\frac{1+z_{e q}}{\Omega_{M o}}\right)^{1 / 4}
$$




\section{CMB Fluctuations}

Adiabatic perturbations (those that fractionally perturb the number densities of photons and matter equally) grow according to

$$
\Delta=\left\{\begin{array}{cr}
\Delta_{D C}\left(\frac{R(t)}{R_{D C}}\right)^{2} & \text { radiation - dominated } \\
\Delta_{e q}\left(\frac{R(t)}{R_{e q}}\right) & \text { matter - dominated }
\end{array}\right.
$$

An estimation of the scale of fluctuations at last scattering is given by

$$
\Delta_{L S}=\left(\frac{R_{L S}}{R_{e q}}\right)\left(\frac{R_{e q}}{R_{D C}}\right)^{2} \Delta_{D C}=\frac{\left(1+z_{D C}\right)^{2}}{\left(1+z_{e q}\right)\left(1+z_{L S}\right)} \Delta_{D C}
$$




\section{Size of Fluctuations}

The energy driving fluctuations during dark energy de-coherence is expected to be given by the cosmological dark energy. This means that the amplitude of relative fluctuations $\delta \rho / \rho$ is expected to be of the order

$$
\Delta_{D C} \equiv\left(\frac{\rho_{\Lambda}}{\rho_{D C}}\right)^{1 / 2}=\frac{R_{D C}}{R_{\Lambda}}
$$

Using the previous equations, this amplitude at last scattering is given by

$$
\Delta_{L S}=\frac{\left(1+z_{D C}\right)^{2}}{\left(1+z_{e q}\right)\left(1+z_{L S}\right)} \frac{R_{D C}}{R_{\Lambda}} \cong \frac{1}{1+z_{L S}} \sqrt{\frac{\Omega_{\Lambda o}}{\left(1-\Omega_{\Lambda o}\right)\left(1+z_{e q}\right)}} \approx 2.6 \times 10^{-5}
$$

where a spatially flat cosmology has been assumed. This estimate is independent of the scale parameter during de-coherence $R_{D C}$, and is of the order observed for the fluctuations in the CMB. Fluctuations in the $\mathrm{CMB}$ at last scattering of this order are consistent with the currently observed clustering of galaxies. 


\section{DE-COHERENCE DURING DUST/PLASMA DOMINATION}

The previous results have demonstrated NO dependency on the energy density during the transition period if de-coherence occurs during radiation domination. For completeness, the amplitude of expected fluctuations if the phase transition occurs during the dust/plasma dominated era is next examined. The acoustic wave has coherent phase information that is transmitted to the $\mathrm{CMB}$ at last scattering. There must have been a significant enough passage of time from the creation of the acoustic wave to the time of last scattering such that peaks and troughs of the various modes should be present $\delta t>D_{s} / v_{s}$, where $D_{s}$ is the distance scale of the longest wavelength (sound horizon), and $v_{s} \sim c / \sqrt{3}$ is the speed of the acoustic wave.

Generally, if the phase transition occurs while the energy density is dominated by dust/plasma, prior to last scattering then the amplitude satisfies

$$
\Delta_{L S} \cong\left(\frac{1+z_{D C}}{1+z_{L S}}\right) \sqrt{\frac{\Omega_{\Lambda o}}{\left(1-\Omega_{\Lambda o}\right)\left(1+z_{D C}\right)^{3}\left(1+\frac{1+z_{D C}}{1+z_{e q}}\right)}}
$$

which varies from $2 \times 10^{-5}$ if the phase transition occurs at radiation dust equality, to $4 \times 10^{-5}$ if it occurs at last scattering. 


\section{Thermalization Scenarios}

- System undergoes a thermal phase transition at de-coherence

- System undergoes an inflationary transition at de-coherence

- System undergoes a purely quantum phase transistion at de-coherence

- System initially classical 


\section{Thermal Expansion}

Assume the cosmology remains a hot radiation dominated thermal system during de-coherence with the microscopic degrees of freedom due to the particle spectrum included.

$$
\begin{aligned}
\rho_{D C}=\frac{8 \pi}{3}\left(M_{P}^{2} \varepsilon^{2}\right) & =g\left(T_{D C}\right) \frac{\pi^{2}}{30}\left(k_{B} T_{D C}\right)^{4} \\
\Rightarrow k_{B} T_{D C} & \cong 1298 \mathrm{GeV}
\end{aligned}
$$

Here $g\left(T_{D C}\right)$ counts the number of degrees of freedom associated with particles of mass $m c^{2}<k_{B} T_{D C}$, and $M_{P}$ is the Planck mass.

This would correspond

to a redshift of

$$
z_{D C} \cong 1.35 \times 10^{16}
$$




\section{Dark Matter Condensation}

If the de-coherence scale corresponds to the evaporation of a dark matter condensate, the mass scale of the dark matter can be determined

$$
\begin{gathered}
z_{D C} \cong 1.35 \times 10^{16} \\
z_{\text {crit }} \cong 1.38 \times 10^{18}\left(\frac{g\left(T_{\gamma_{o}}\right)}{g\left(T_{\text {crit }}\right)}\right)^{1 / 3} g_{m_{D M}}^{2 / 3}\left(\frac{m_{D M} c^{2}}{G e V}\right)^{5 / 3} \\
\Rightarrow m_{D M} c^{2} \cong \frac{0.107 G e V}{g_{m_{D M}}^{2 / 5}}
\end{gathered}
$$




\section{Inflationary Transition}

If the energy density at de-coherence is the inflationary dark energy density, this then defines the de-Sitter scale of inflation

$$
\rho_{\Lambda_{i}}=\frac{\Lambda_{i}}{8 \pi G_{N}}=\frac{3}{8 \pi} M_{P}^{2} \varepsilon^{2} \Rightarrow \Lambda_{i}=\frac{3}{R_{\varepsilon}^{2}}
$$

Scale associated with present

De Sitter entropy

De Sitter temperature at de-coherence cosmological constant defines inflationary scale

$$
\begin{gathered}
S_{\varepsilon}=\frac{A_{\varepsilon}}{4 G_{N}}=\pi\left(\frac{M_{P} c^{2}}{\varepsilon}\right)^{2} k_{B} \\
k_{B} T_{\varepsilon}=\frac{1}{2 \pi R_{\varepsilon}}=\frac{\varepsilon}{2 \pi}
\end{gathered}
$$

This means that the scale of the horizon temperature during inflation is comparable to the dark energy today (ie the thermal energy from the previous expansion corresponds to the dark energy of the present expansion). We indeed expect this thermal energy to drive the initial fluctuations! 


\section{What's So Special about $\varepsilon$ ?}

Thus far, ANY value for $\varepsilon$ will give the observed scale for the amplitude of fluctuations in the CMB. The observed scale of the dark energy might just be a random fluctuation. One might alternatively expect microscopic physics to fix the particular scale as a quantum phase transition associated with a UV momentum or mass scale for the gravitational modes.

$$
\rho_{\Lambda_{i}}=\frac{1}{V} \sum_{P_{I R}}^{P_{U V}} \frac{1}{2} \hbar c P_{j} \cong\left(\frac{1}{2 \pi}\right)^{2} \frac{1}{4} P_{U V}^{4} \equiv m_{U V}^{4} \quad \begin{gathered}
\text { Space filling until phase } \\
\text { transition at de-coherence } \\
m_{U V} \cong 3160 \mathrm{GeV}
\end{gathered}
$$

At de-coherence, actual masses $m$ separated by their Compton wavelengths can produce this energy density. Such particles would have coherence distances satisfying

$$
8(\Delta X)^{2} \cong R_{\varepsilon} \lambda_{m} \quad, \quad \frac{(\Delta P)^{2}}{2 m} \cong \mathcal{E} \quad \text { Independent of coupling! }
$$




\section{Input of Microscopic Scale}

- $\Delta \mathrm{X}$ and $\lambda_{\mathrm{m}}$ are microscopic scales.

- Critical density of cosmology must be reached before microscopic mass scale can "de-cohere". Prior to reaching this density there is no space to de-cohere into.

- Many mass units are still within the coherence distance, leading one to suspect a macroscopic quantum system

- Expected equation of state

$$
E=\frac{1}{8} N^{2} \varepsilon=m_{U V}^{4} \mathcal{E}^{3} \Rightarrow \frac{\left\langle(\delta \rho)^{2}\right\rangle}{\langle\rho\rangle^{2}}=3 \frac{\rho_{\Lambda}}{\rho_{D C}}
$$




\section{Loss of IR Modes}

Assume spatially flat FRW Cosmology, and a harmonic oscillator

$$
\begin{gathered}
\mathcal{L}=\frac{1}{2}\left(-g^{\mu \nu} \partial_{\mu} \psi \partial_{\nu} \psi-\omega^{2} \psi^{2}\right) ; W=\int \sqrt{-g} \mathfrak{L} d^{4} x \\
\ddot{\psi}-\frac{1}{R^{2}} \nabla^{2} \psi+\omega^{2} \psi+3\left(\frac{\dot{R}}{R}\right) \dot{\psi}=0
\end{gathered}
$$

Expect significant damping if frequencies are of Hubble scale

Similarly, using quantum measurability arguments, quantized energy scale $\mathrm{E}_{\mathrm{IR}}$ cannot satisfy uncertainty relation prior to time

$$
\dot{R}_{I R}=c, R_{I R} \equiv \frac{\hbar c}{E_{I R}}, t_{I R}=\frac{R_{I R}}{2 c} \longleftarrow \quad \begin{aligned}
& \text { assuming radiation } \\
& \text { dominated epoch }
\end{aligned}
$$




\section{Early Time Thermal Behavior}

Friedmann-LeMaitre+Quantum Uncertainty imply a loss of IR energy modes as the cosmology becomes denser going back in time. If there is a UV gravitating mode (needed for finite vacuum energy density in terms of interaction quanta), then the early time thermal behavior should change from the $\mathrm{T}^{4}$ behavior far from the cutoff.

Expect $\left(\frac{P_{U V}}{P_{\Lambda}}\right)^{4}=\frac{8 \pi}{3}\left(\frac{M_{P} c^{2}}{\varepsilon}\right)^{2}=\frac{8 \pi^{2}}{9} N^{2}$

$$
\sum_{I R}^{U V} \doteq \frac{V}{(2 \pi \hbar)^{3}} \int_{P_{I R}}^{P_{U V}} 4 \pi P^{2} d P
$$

As the IR cutoff approaches the UV cutoff, the thermal energy should behave as

$$
U=U_{I R}+g(T) \frac{4 \pi V}{(2 \pi \hbar)^{3}}\left(P_{U V} c\right)^{4} \frac{1-\frac{P_{I R}}{P_{U V}}}{e^{P_{U V} c / k_{B} T}-1}
$$

where $\mathrm{U}_{\mathrm{IR}}$ might have macroscopic occupation. Stephan's Law is expected to no longer hold. 


\section{Alternative Scenarios}

In any such scenario, we expect independent (Gaussian) fluctuations to develop in each mode as expansion proceeds.

Scenario 1: Low temperature behavior

Macroscopic occupation in IR mode, reheating of entropy as modes become available

Scenario 2: High temperature bosonic system

$$
\begin{aligned}
& U=U_{I R}+g(T) \frac{4 \pi V}{(2 \pi \hbar c)^{3}}\left(P_{U V} c\right)^{4}\left(1-\frac{P_{I R}}{P_{U V}}\right) \frac{k_{B} T}{P_{U V} c} \\
& S=S_{I R}+g(T) \frac{4 \pi V}{(2 \pi \hbar c)^{3}}\left(P_{U V} c\right)^{4}\left(1-\frac{P_{I R}}{P_{U V}}\right) \frac{k_{B}}{P_{U V} c} \log \left(\frac{T}{T_{I R}}\right)
\end{aligned}
$$




\section{Black Hole Evaporation}

Expected rate of evaporation

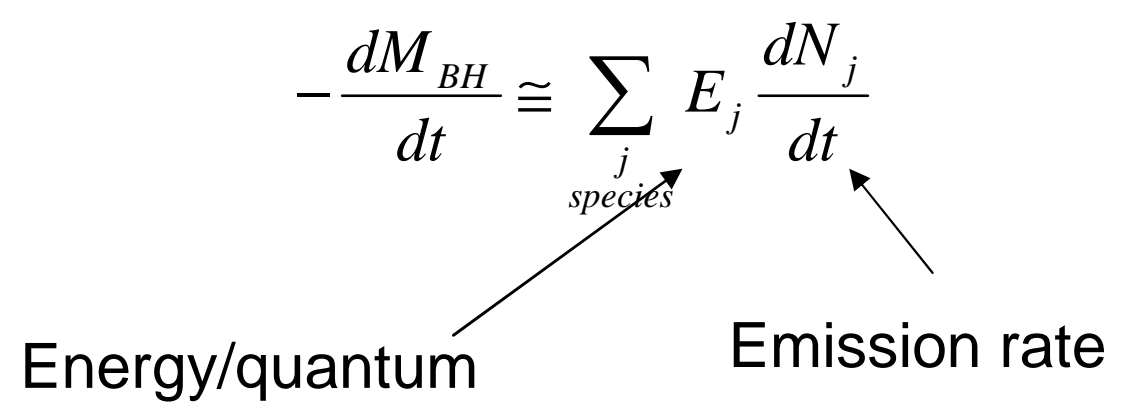

"Thermal” phase space

$$
k_{B} T_{H}=\frac{1}{8 \pi M G_{N}}=\frac{1}{8 \pi} \frac{\left(M_{P} c^{2}\right)^{2}}{M c^{2}} \Rightarrow E_{j} \cong k_{B} T_{H}
$$

Most emitted quanta will be s-wave, and the barrier height is proportional to the temperature. This means one expects about 1 quantum per unit Rindler time, which in Schwarzschild time results in

$$
\frac{d N_{j}}{d t} \cong f g_{j} \frac{c}{R_{S}} \Theta\left(k_{B} T_{H}-m_{j} c^{2}\right)
$$

Black hole will not begin emitting particles $m_{j}$ until its Hawking temperature is sufficient: $k_{B} T_{H}>m_{j} c^{2}$

$$
\boldsymbol{M}_{j} \equiv \frac{1}{8 \pi} \frac{M_{P}^{2}}{m_{j}}
$$




\section{Number of Low Mass Quanta}

$$
\begin{aligned}
& M_{B H}\left(t_{j}<t<t_{j+1}\right) \cong\left(\boldsymbol{M}_{j}^{3}-\frac{3 f}{16 \pi} g\left(m_{j}\right) M_{P}^{4} \frac{c^{2}}{\hbar}\left(t-t_{j}\right)\right)^{1 / 3} \\
& N_{j}\left(t_{j}<t<t_{j+1}\right) \cong 4 \pi \frac{g_{j}}{g\left(m_{j}\right)}\left\{\left(\frac{\boldsymbol{M}_{j}}{M_{P}}\right)^{2}-\left(\left(\frac{\boldsymbol{M}_{j}}{M_{P}}\right)^{3}-\frac{3 f}{16 \pi} g\left(m_{j}\right) \frac{M_{P} c^{2}}{\hbar}\left(t-t_{j}\right)\right)^{2 / 3}\right\}
\end{aligned}
$$

If the subscript $F$ represents the highest mass particle in the spectrum, the total such particles emitted is

$$
N_{F}^{\text {Total }} \cong 4 \pi \frac{g_{F}}{g\left(m_{F}\right)}\left(\frac{\boldsymbol{M}_{F}}{M_{P}}\right)^{2}=\frac{1}{16 \pi^{2}} \frac{g_{F}}{g\left(m_{F}\right)}\left(\frac{M_{P}}{m_{F}}\right)^{2}
$$

Similarly, the number of zero mass quanta emitted is

$$
\begin{aligned}
& N_{0}^{\text {Total }} \cong \\
& 4 \pi\left\{\left(\frac{M_{B H}}{M_{P}}\right)^{2}+\sum_{j=0}^{F-1} g_{0}\left(\frac{1}{g\left(m_{j+1}\right)}-\frac{1}{g\left(m_{j}\right)}\right) \frac{1}{64 \pi^{2}}\left(\frac{M_{P}}{m_{j+1}}\right)^{2}+\frac{1}{64 \pi^{2}} \frac{g_{0}}{g\left(m_{j}\right)}\left(\frac{M_{P}}{m_{F}}\right)^{2}\right\}
\end{aligned}
$$




\section{Ratio of Evaporated Particle Number}

If initial thermalization into the particle spectrum proceeds primarily through black hole evaporations, ratios of gravitating only masses should be fixed at this stage. If dark matter only gravitates, the number should correspond to

$$
\frac{N_{D M}}{N_{\gamma}} \cong 3.70 \times 10^{-9}\left(\frac{G e V}{m_{D M} c^{2}}\right)
$$

If dark matter is particulate, this number should be generated through some sort of gravitational dynamics (ie due to its gravitational coupling $\mathrm{G}_{\mathrm{N}} \mathrm{m}_{\mathrm{DM}}{ }^{2}$ ) during this de-coherence phase. 


\section{Quantum Early Universe}

Examine symmetry breaking in early universe

$$
L=-\frac{1}{4} F^{\mu v} F_{\mu \nu}-\frac{1}{2}\left(D_{\mu} \Phi_{a}\right) g^{\mu \nu}\left(D_{\nu} \Phi_{a}\right)+\frac{1}{4} m_{\Phi}^{2} \Phi_{a}^{2}-\frac{1}{8} f^{2} \Phi_{a}^{4}
$$

Classical solution, gauge fields vanish $A=0$

$$
\begin{aligned}
& \left.k \Phi_{b}\right\rangle \mid=\frac{m_{\Phi}}{f}=\frac{m_{B}}{e} \\
& \text { Higgs } \\
& \text { Vector Boson }
\end{aligned}
$$

$$
T_{0}^{0}=\frac{1}{8}\left(\frac{m_{\Phi}^{2}}{f}\right)^{2}
$$

Can argue this as density scale of de-coherence $\rho_{D C}$

Choosing electro-weak symmetry restoration estimates of the early 90's, Ed Jones predicted a cosmological constant with $\Omega_{\Lambda} \sim 0.6$ BEFORE this idea was fashionable! 


\section{Discussion and Conclusion}

- We have shown that de-coherence driven by dark energy gives the expected order for the amplitude of fluctuations in the $\mathrm{CMB}$ radiation.

- We generally expect some form of cosmological quantum coherence at a scale far from the Planck scale, implying space-like correlations and phase coherence.

- We expect microscopic luminal interactions to differentiate amongst pre-coherent cosmologies, all of which give the same amplitude of fluctuations, fixing a cosmological constant for sub-luminal scale expansion. For the observed dark energy density, the cosmological density at decoherence turns out to be given by the electro-weak symmetry scale.

- Spatial flatness is required for observed structure formation using the relevant microscopic scale in the FL equation.

- Some form of microscopic manifestation of gravitational physics is expected on the TeV energy scale. 


\section{Acknowledgements}

The author gratefully acknowledges useful discussions with the following:

Stephon Alexander, James Bjorken, Stan Brodsky, E.D.

Jones, L.H. Kauffman, T.W.B. Kibble, Walter Lamb, H.

Pierre Noyes, Michael Peskin, and Lenny Susskind.

This work was supported through the visiting scholars program of $\mathrm{H}$. Pierre Noyes 


\section{REFERENCES}

- $\quad$ For more on this presentation, see

- J.V. Lindesay and H.P.Noyes, “A Calculation of Cosmological Scale from Quantum Coherence", astro$\mathrm{ph} / 040753543$ pages (2004).

- J.V. Lindesay and H.P.Noyes, "Cosmic Microwave Background Fluctuation Amplitude from Dark Energy De-Coherence", astro-ph/0410217 9 pages (2004).

- J.V. Lindesay, H.P.Noyes, and E.D. Jones, "CMB Fluctuation Amplitude from Dark Energy Partitions", astro-ph/0412477 7 pages (2004).

- A recent review article that incorporates some of these views is give by T. Padmanabhan, "Understanding Our Universe: Current Status and Open Issues", gr-qc/0503107, 30 pages

- Quantum coherence of gravitating systems was demonstrated in A.W.Overhauser and R.Colella, Phys.Rev.Lett. \{lbf 33$\}, 1237$ (1974); R.Colella, A.W.Overhauser and S.A.Werner, Phys.Rev.Lett. \{ \bf $34\}, 1472$ (1975).

- $\quad$ For more on Casimir and zero-point motions, see

- H.B.G.Casimir, Proc.K.Ned.Akad.Wet. 51, 793 (1948)

- $\quad$ E.M. Lifshitz, Soviet Phys. JETP 2, 73 (1956).

- I.D. Dzyaloshinskii, E.M. Lifshitz, and I.P. Pitaevskii, Soviet Phys. Usp. 4, 153 (1961).

- I.D. Landau and E.M. Lifshitz, Itextit\{Electrodynamics of Continuous Media\}, pp368-376 (Pergamon, Oxford, 1960)

- $\quad$ D. Kleppner, "With Apologies to Casimir", Physics Today, (Oct. 1990), pp 9-11.

- For more on cluster de-composition and de-coherence, see

- $\quad$ M.Alfred, P.Kwizera, J.V.Lindesay and H.P.Noyes, “A Non-Perturbative, Finite Particle Number Approach to Relativistic Scattering Theory, hep-th/0105241, Found.Phys.34:581-616(2004).

- J.V.Lindesay, A.Markevich, H.P.Noyes, and G.Pastrana, "Self-Consistent Poincare-Invariant and Unitary 3-Particle Theory", Phys.Rev.D.33,2339-2349(1986). 